\title{
A Distributed Control Scheme of Microgrids in Energy Internet Paradigm and Its Multi-Site Implementation
}

\author{
Yu Wang, Member, IEEE, Tung Lam Nguyen, Mazheruddin H. Syed, Member, IEEE, Yan Xu, \\ Member, IEEE, Efren Guillo-Sansano, Member, IEEE, Van-Hoa Nguyen, Graeme Burt, Member, \\ IEEE, Quoc-Tuan Tran, Member, IEEE, and Raphael Caire, Member, IEEE.
}

\begin{abstract}
Internet-of-Things concepts are evolving the power systems to the Energy Internet paradigm. Microgrids (MGs), as the basic element in an Energy Internet, are expected to be controlled in a corporative and flexible manner. This paper proposes a novel distributed control scheme for multi-agent systems (MASs) governed MGs in future Energy Internet. The control objectives are frequency/voltage restoration and proportional power sharing. The proposed control scheme considers both intra and inter MASs interactions, which offers group plug-and-play capability of distributed generators (DGs). The stability and communication delay issues in the control framework are analysed. A multi-site implementation framework is presented to explain the agent architecture as well as data exchange in local area networks and the cloud server. Then a cyber hardware-in-the-loop $(\mathrm{C}$-HiL) experiment is conducted to validate the proposed control method with multi-site implementation. The experimental results prove the effectiveness and application potentials of the proposed approach.
\end{abstract}

Index Terms - Energy Internet, multi-agent system, distributed control, microgrids, hardware-in-the-loop.

\section{INTRODUCTION}

$I^{\mathrm{N}}$ NTERNET of Things (IoT) is a paradigm that bridges a variety of real, digital and virtual devices through information networks into smart environments and spans across domains such as energy, transportation, cities, etc [1]. Energy Internet, as a revolutionary vision of smart grids, can be a typical IoT application in power and energy industry [2], [3]. The Energy Internet comprises various components and techniques that can be summarized into three categories: (i) power systems; (ii) communication systems, (iii) control algorithms. The cross-disciplinary nature of the Energy Internet has brought forward new challenges and opportunities, which require extensive research and validation.

The microgrids (MGs) serve as the basic building blocks in the Energy Internet, capable of operating in both islanded and grid-connected mode [4], [5]. The droop based primary control is used for autonomous power-sharing among distributed

This work is supported in part by the European Liaison on Electricity Committed Towards long-term Research Activity (ELECTRA) Integrated Research Program (IRP), in part by the ERIGrid program, and in part by Ministry of Education (MOE), Republic of Singapore, under grant AcRF TIER 1 2019-T1-001-069 (RG75/19). Y. Xu's work is supported by Nanyang Assistant Professorship from Nanyang Technological University, Singapore.

Y. Wang and Y. Xu are with Nanyang Technological University, Singapore 639798 (e-mail: wang_yu@ntu.edu.sg, xuyan@ntu.edu.sg). generators (DGs). The secondary control of islanded MGs achieves the frequency/voltage restoration while maintaining accurate power-sharing among DGs [6], [7]. The tertiary control is usually responsible for the optimal operation of MGs [8], [9]. In a hierarchical control scheme, the tertiary control determines the optimal dispatch values based on the load and renewable forecast. Within the dispatch intervals (e.g. every 15 minutes), the primary and secondary control operate to share the real-time power deviations from the dispatch values.. In literature, distributed consensus algorithms based secondary control and distributed optimization algorithms based tertiary control have garnered much attention due to their enhanced flexibility and resilience over centralized control [10], [11]. The realization of the distributed algorithms relies on multi-agent systems (MASs), where multiple agents/subsystems interact with each other via sparse communication networks [12].

In the Energy Internet, many practical issues and challenges emerge with the deployment of MASs techniques into MGs. This paper targets to provide potential solutions for the following three scenarios: (i) The distributed controllers may neither be located at the same location as DGs nor have a proprietary communication network. The remote control of MGs via the Internet taking communication latency into consideration is required. (ii) For MGs governed by MASs, each agent or sub-MAS can be practically owned by different stakeholders which could cooperate together or work independently. A flexible control framework with plug-andplay capability is needed. (iii) With the advancements in IoT and renewable technology, the number of controllable units in MGs are dramatically increasing. The scalability of any distributed control framework to withstand increasing numbers of DGs is a problem worthy of exploration.

In state-of-the-art, various control algorithms for distributed secondary control have been proposed, such as optimal control [13], finite-time control [14], event-triggered control [15] and data-driven methods [16]. Their objectives are to improve the control performance from different aspects, such as dynamic

T. L. Nguyen, and R. Caire are with University Grenoble Alpes, G2Elab, F38000 Grenoble, France.

M. H. Syed, E. Guillo-Sansano and G. M. Burt are with the Institute for Energy and Environment, University of Strathclyde, Glasgow, UK.

Q. T. Tran and V. H. Nguyen are with Alternative Energies and Atomic Energy Commission (CEA), National Institute for Solar Energy (INES), France. 
performance [13], convergence speed [14], communication efficiency [15], and robustness against uncertainty [16]. In addition, the time delay in communication channels as an unneglectable issue has been further considered in both controller design and stability analysis [17], [18]. In the context of Energy Internet, a distributed control of DGs in gridconnected MGs is proposed in [19], and an event-triggered hybrid control based on a MAS is proposed in [20].

From the literature, two important research gaps have been identified. Firstly, the distributed control schemes for MASs governed MGs in Energy Internet have not been investigated. This motivates us to provide a new methodology which enables the group plug-and-play feature, such that MGs with multiple MASs owned by different stakeholders can be flexibly controlled. Secondly, the validation of distributed control algorithms is mainly based on simulations [13]-[16], [19] and [20] or one-site experiment without realistic communications [17], [18]. Although experimental study for MG research has been conducted for many years, the validation and design involving real communication networks with multi-site realization are still in the beginning stage. In [21], a three-level coordinated voltage/var control scheme is validated with power hardware-in-the-loop (HiL) test and cable commutations among distributed controllers. In [22] and [23], multi-site cosimulation platforms to emulate the virtual integration of power systems are proposed, but the implementation of distributed controllers on MASs is not included.

To fill the gap between theoretical research and hardware implementation, this paper presents a new distributed control scheme and its multi-site implementation, which enables the remote control of islanded MGs in Energy Internet. The control and implementation architecture allow agents in MASs to flexibly control MGs through cloud services. The ownership of DGs/MGs can be changed by allowing or denying cloud data access to agents. A multi-site cyber hardware-in-the-loop (CHiL) test has been accomplished by Nanyang Technological University (NTU) in Singapore, University of Strathclyde in UK, and University Grenoble Alpes (UGA) in France. The contributions of this paper are summarized as follows:

- First, the emerging problem of MASs governed MGs in Energy Internet is introduced and studied in this paper, which was rarely reported by previous research.

- Second, a distributed secondary control of MGs enabling the group plug-and-play feature is proposed by considering the interaction within and among multiple MASs with different ownership.

- Third, a multi-site implementation framework of the proposed control method via multi-agent systems and cloud servers is introduced.

- Lastly, a multi-lab joint C-HiL experiment is conducted to validate the effectiveness and scalability of the proposed control framework.

\section{PRELIMINARIES}

\section{A. MGs in Energy Internet}

In this paper, it is considered that DGs have the control and communication agents within the realm of Energy Internet, as shown in Fig. 1. The physical entities of a typical MG comprise of inverter-interfaced DGs, (such as photovoltaics (PVs), wind turbines (WTs), energy storage systems), diesel generators, static and dynamic loads [5]. The DGs operating in maximum power point tracking mode (such as PVs and WTs) can be modelled as negative power loads [24]. Other dispatchable DGs in the MG are controlled by the proposed framework where each DG is governed by one agent of the MAS. The agents in MASs communicate through local area networks (LANs) and have access to the Internet to enable remote control of MGs via cloud servers. In Energy Internet, each DG/MG can be owned by the different stakeholders, and their controllers on agents/MAS may be far away from the MG entities. It is also expected that the number of DGs and agents in MGs can be online changed, so a distributed, remote, flexible control and implementation framework is required.

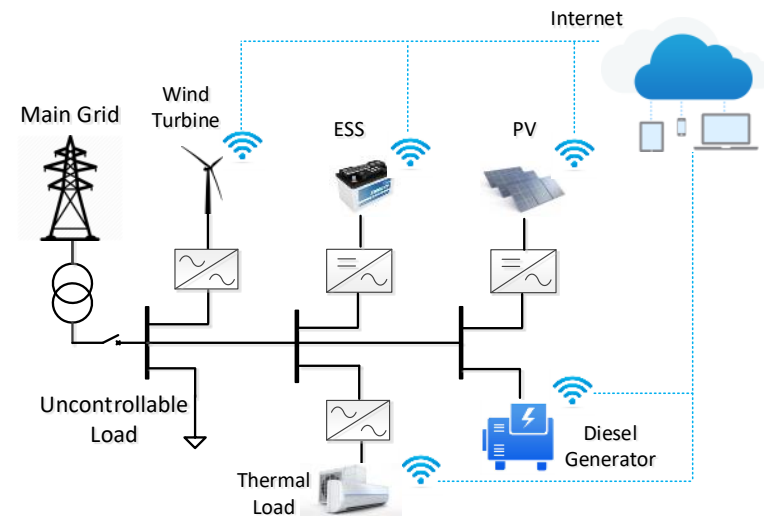

Fig. 1. The architecture of MGs in Energy Internet.

\section{B. Problem Description}

This paper considers that the MG has $N$ controllable DGs (indexed by $i=1,2, \ldots, N$.). The electrical network of MGs is represented by a complex-weighted graph $\mathcal{T}=\left(\mathcal{V}_{\mathcal{T}}, \mathcal{E}_{\mathcal{T}}\right)$, where the nodes $\mathcal{V}_{\mathcal{T}}=\left\{v_{1}, v_{2}, \ldots, v_{N}\right\}$ represent the buses (DGs), and the edges $\mathcal{E}_{\mathcal{T}} \subseteq \mathcal{V}_{\mathcal{T}} \times \mathcal{V}_{\mathcal{T}}$ represent the line connections. Considering the inductive output impedance of DGs and the power angle differences are small [8], [12], the basic principle of droop control (i.e., $P$ versus $\omega$ and $Q$ versus $V$ ) can be represented as follows:

$$
\begin{aligned}
\omega_{i} & =\omega_{i}^{\mathrm{nom}}-m_{i}^{P} P_{i} \\
V_{i} & =V_{i}^{\mathrm{nom}}-m_{i}^{Q} Q_{i}
\end{aligned}
$$

where $\omega_{i}^{\text {nom }}$ and $V_{i}^{\text {nom }}$ are the nominal set-points of frequency and voltage amplitude, respectively. $m_{i}^{P}$ and $m_{i}^{Q}$ are droop coefficients, which are commonly selected based on the maximum output power as $m_{i}^{P}=\Delta \omega / P_{i}^{\max }$ and $m_{i}^{Q}=\Delta V / Q_{i}^{\max }$, where $\Delta \omega$ and $\Delta v$ are allowable frequency and voltage deviations of the MG. The voltage magnitude is calculated by $V_{i}=\sqrt{\left(v_{i}^{d}\right)^{2}+\left(v_{i}^{q}\right)^{2}}$ with d-axis and q-axis voltages $v_{i}^{d}$ and $v_{i}^{q}$. As the reference frame of the voltage magnitude is aligned to d-axis, thus $V_{i}=v_{i}^{d}, v_{i}^{q}=0$.

The nonlinear dynamics of each DG can be represented by a differential equation with 13 state variables, which is not given 
here for brevity [25]. Based on the feedback linearization, the secondary control of droop controlled DGs in islanded MGs can be formulated as follows [13], [15], [26]:

$$
\begin{aligned}
& \dot{\omega}_{i}=\dot{\omega}_{i}^{\text {nom }}-m_{i}^{P} \dot{P}_{i}=u_{i}^{\omega} \\
& \dot{V}_{i}=\dot{V}_{i}^{\text {nom }}-m_{i}^{Q} \dot{Q}_{i}=u_{i}^{V}
\end{aligned}
$$

The problem of accurate power-sharing control can be formulated as $m_{i}^{P} \dot{P}_{i}=u_{i}^{P}, m_{i}^{Q} \dot{Q}_{i}=u_{i}^{Q}$. Then the nominal setpoints $\omega_{i}^{\text {nom }}$ and $V_{i}^{\text {nom }}$ are determined by the secondary control as follows:

$$
\begin{aligned}
& \omega^{\mathrm{nom}}=\int\left(\dot{\omega}_{i}+m_{i}^{P} \dot{P}_{i}\right) d t=\int\left(u_{i}^{\omega}+u_{i}^{P}\right) d t \\
& V^{\mathrm{nom}}=\int\left(\dot{V}_{i}+m_{i}^{P} \dot{Q}_{i}\right) d t=\int\left(u_{i}^{V}+u_{i}^{Q}\right) d t
\end{aligned}
$$

As observed from (5) and (6), the secondary control inputs of $\boldsymbol{u}_{i}^{\omega}$ and $\boldsymbol{u}_{i}^{P}$ control $\omega^{\text {nom }}$, while the secondary control inputs of $u_{i}^{\omega}$ and $u_{i}^{Q}$ control $V^{\mathrm{nom}}$.

The control objectives of the proposed distributed secondary control are presented as follows:

1. The frequency restoration of MGs

$$
\lim _{t \rightarrow \infty}\left|\omega_{i}(t)-\omega^{r e f}\right|=0, \forall i=1,2, \ldots, N
$$

2. The voltage restoration of MGs

$$
\lim _{t \rightarrow \infty}\left|V_{i}(t)-V^{r e f}\right|=0, \forall i=1,2, \ldots, N
$$

3. The accurate real power-sharing among DGs

$$
\lim _{t \rightarrow \infty}\left|m_{i}^{P} P_{i}(t)-m_{i}^{P} P_{j}(t)\right|=0, \forall i \neq j
$$

where $\omega^{r e f}$ and $V^{r e f}$ are the frequency and voltage reference values. These objectives are met by adjusting control inputs $u_{i}^{\omega}$, $u_{i}^{P}$ and $u_{i}^{V}$ of each agent. Due to the trade-off relationship between voltage restoration and accurate reactive power sharing among DGs, only voltage control is considered in this paper, which is also adopted in [13]-[15].

Remark 1: According to (9), the power ratio $m_{i}^{P} P_{i}(t)$ among DGs will be equalized in steady-state. Together with the definition of droop coefficient $m_{i}^{P}=\Delta \omega / P_{i}^{\max }$, the real power among DGs are shared as:

$$
\frac{P_{i}}{P_{j}}=\frac{m_{j}^{P}}{m_{i}^{P}}=\frac{\Delta \omega / P_{j}^{\max }}{\Delta \omega / P_{i}^{\max }}=\frac{P_{i}^{\max }}{P_{j}^{\max }}
$$

Eq. (10) means the real power among DGs are shared proportionally to their power ratings and inversely to their droop coefficients in steady-state.

\section{Communication Networks of MASs}

The communication network of a MG with $N$ agents is depicted by a graph: $\mathcal{G}=\left(\mathcal{V}_{\mathcal{G}}, \mathcal{E}_{\mathcal{G}}\right)$ with a set of nodes $\mathcal{V}_{\mathcal{G}}=\left\{v_{1}, v_{2}, \ldots, v_{N}\right\}$ and a set of edges $\mathcal{E}_{\mathcal{G}} \subseteq \mathcal{V}_{\mathcal{G}} \times \mathcal{V}_{\mathcal{G}}$. The nodes in graph $\mathcal{G}$ (agents) are one to one corresponding to nodes in graph $\mathcal{T}$ (DGs). The edges in $\mathcal{G}$, which represent communication links for data exchange, can be different from the electrical connection in $\mathcal{T}$. The set of neighbors of $i$ th node in $\mathcal{G}$ is represented by $N_{i}=\left\{v_{j} \in \mathcal{V}_{\mathcal{G}}:\left(v_{i}, v_{j}\right) \in \mathcal{E}_{\mathcal{G}}\right\}$. The adjacency matrix is represented by $A=\left[a_{i j}\right] \subseteq \mathbb{R}^{n \times n}$. The element $a_{i j}$ represents the information exchanged between agents $i$ and $j$, where $a_{i j}=1$ if agents $i$ and $j$ are connected with an edge $\left(v_{i}, v_{j}\right) \in \mathcal{E}_{\mathcal{G}}$, otherwise, $a_{i j}=0$. The Laplacian matrix is represented by $L=\left[l_{i j}\right] \subseteq \mathbb{R}^{n \times n} \quad$ with each element $l_{i j}=\sum_{i=1}^{n} a_{i j}-a_{i j}$. The pinning matrix is represented by $G=\operatorname{diag}\left[g_{i}\right] \subseteq \mathbb{R}^{n \times n}$, and $g_{i}=1$ if this agent/DG has access to references $\omega^{\text {ref }}$ and $V^{\text {ref }}$, otherwise $g_{i}=0$.

\section{PROPOSED CONTROL FRAMEWORK}

\section{A. Overview}

An overview of the proposed control framework and its CHiL implementation is depicted in Fig. 2. The left part of Fig. 2 shows the control structure, where $N$ DGs in the MG are governed by $N$ agents in MASs. Particularly, the agents in MASs are divided into $m$ groups with $n$ agents in each group, which is similar to the condition that each MAS is owned by different stakeholders. A scalable and flexible distributed control scheme is proposed considering intra and inter MAS interactions. In each control process of the MG, the data packet of electrical parameters $\left\{\omega_{i}, V_{i}, m_{i}^{P} P_{i}\right\}$ and the data packet of secondary control signals $\left\{\omega_{i}^{\text {nom }}, V_{i}^{\text {nom }}\right\}$ are exchanged via the cloud server.

The right part of Fig. 2 shows the implementation setup. An Opal-RT, RPis, and Redis based C-HiL platform is built to validate the distributed controller design. The physical entity of islanded MGs is implemented in real-time on OPAL-RT. The MAS and its associated distributed controllers are developed on hardware embedded systems - Raspberry Pis (RPis) [27]. The software environment of the MAS platform is developed using Google remote procedure call (gRPC) framework [28]. The data exchange between MASs and MGs is realized via the cloud server on Redis [29]. As shown in Fig.2, this cloud-based control framework links laboratories in UK, France, and Singapore, in real-time respectively. In the following sections, the design of distributed secondary controllers and their realization on the MAS platform will be introduced.

\section{B. Controller Design}

The purpose of this section is to propose a control framework with good scalability and flexibility in Energy Internet. Various distributed secondary control methods have been investigated in [13]-[19], and here a widely used linear control protocol for each DG as in [26] is adopted. For each group/MAS with $n$ agents, the control protocol is given as follows:

$$
\begin{array}{r}
u_{i}^{\omega}(t)=k_{i}^{\omega}\left[\sum_{j=1}^{N} a_{i j}\left(\omega_{j}(t)-\omega_{i}(t)\right)+g_{i}\left(\omega^{r e f}-\omega_{i}(t)\right)\right] \\
u_{i}^{V}(t)=k_{i}^{V}\left[\sum_{j=1}^{N} a_{i j}\left(V_{j}(t)-V_{i}(t)\right)+g_{i}\left(V^{r e f}-V_{i}(t)\right)\right] \\
u_{i}^{p}(t)=k_{i}^{p}\left[\sum_{j=1}^{N} a_{i j}\left(p_{j}(t)-p_{i}(t)\right)\right]
\end{array}
$$

where $i, j \in\{1,2 \ldots, n\}, p_{i}=m_{i}^{P} P_{i}$ for simplicity, the control gains $k_{i}^{\omega}, k_{i}^{V}$ and $k_{i}^{p}$ are all greater than zero. 


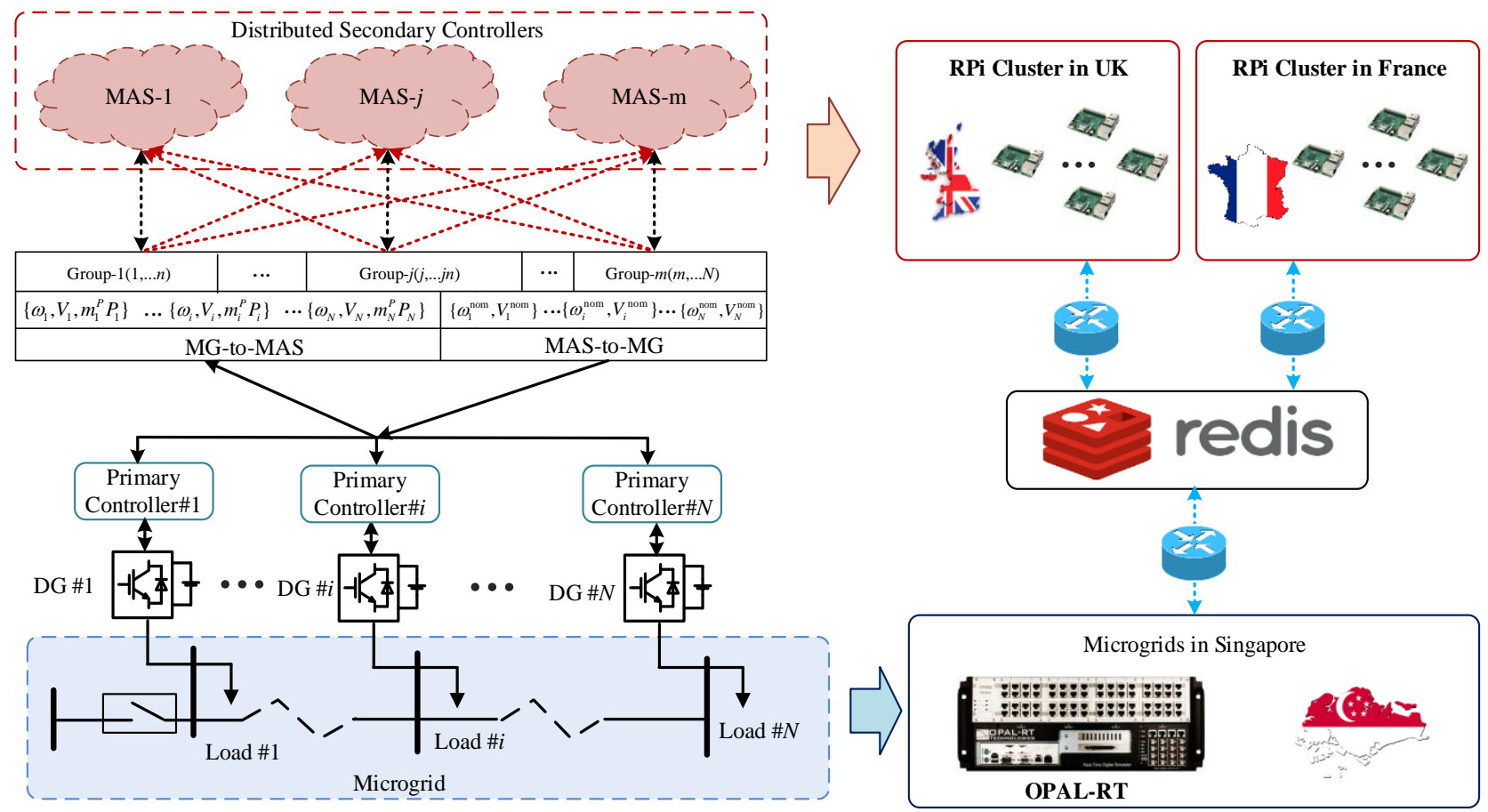

Fig. 2. The framework of proposed distributed secondary control via cloud server.

In compact form, the above equations (11)-(13) can be represented by:

$$
\begin{gathered}
u^{\omega}=k^{\omega}\left(-L \omega+G\left(\omega^{r e f} 1_{n \times 1}-\omega\right)\right) \\
u^{V}=k^{V}\left(-L V+G\left(V^{r e f} 1_{n \times 1}-V\right)\right) \\
u^{p}=-k^{P} L p
\end{gathered}
$$

where the vectors $u^{\omega}=\left[u_{1}^{\omega}, \ldots u_{n}^{\omega}\right]^{T} \quad, \quad u^{V}=\left[u_{1}^{V}, \ldots u_{n}^{V}\right]^{T}$ $u^{p}=\left[u_{1}^{p}, \ldots u_{n}^{p}\right]^{T} k^{\omega}=\operatorname{diag}\left(k_{1}^{\omega}, \ldots k_{n}^{\omega}\right) \quad, \quad k^{V}=\operatorname{diag}\left(k_{1}^{V}, \ldots k_{n}^{V}\right)$, $k^{P}=\operatorname{diag}\left(k_{1}^{P}, \ldots k_{n}^{P}\right)$.

Then all the control inputs from each group/MAS can be represented in matrix form as:

$$
\begin{aligned}
& {\left[\begin{array}{l}
u^{\omega} \\
u^{V} \\
u^{p}
\end{array}\right]=-\left[\begin{array}{ccc}
k^{\omega} & 0 & 0 \\
0 & k^{V} & 0 \\
0 & 0 & k^{p}
\end{array}\right] \underbrace{\left[\begin{array}{ccc}
L & 0 & 0 \\
0 & L & 0 \\
0 & 0 & L
\end{array}\right]}\left[\begin{array}{l}
\omega \\
V \\
p
\end{array}\right]} \\
& u \subseteq \mathbb{R}^{3 n \times 1} \underbrace{0}_{K \subseteq \mathbb{R}^{3 n \times 3 n}} \underbrace{0}_{\tilde{L} \subseteq \mathbb{R}^{3 n 3 n}} \quad \mathbb{R}_{\mathbb{R}^{3 n \times 1}} \\
& +\underbrace{\left[\begin{array}{ccc}
k^{\omega} & 0 & 0 \\
0 & k^{V} & 0 \\
0 & 0 & k^{p}
\end{array}\right]}_{K \subseteq \mathbb{R}^{3 \times \times 3 n}} \underbrace{\left[\begin{array}{ccc}
G & 0 & 0 \\
0 & G & 0 \\
0 & 0 & 0
\end{array}\right]}_{\tilde{G} \subseteq \mathbb{R}^{3 \times \times 3 n}} \underbrace{\left[\begin{array}{c}
\omega^{r e f} 1_{N \times 1}-\omega \\
V^{r e f} 1_{N \times 1}-V \\
0_{N \times 1}-P
\end{array}\right]}_{x^{r e f}-x}
\end{aligned}
$$

For simplicity, (17) can be represented as:

$$
u=K\left[-\tilde{L} x+\tilde{G}\left(x^{r e f}-x\right)\right]
$$

where $u, x, x^{r e f}, K, \tilde{L}$ and $\tilde{G}$ are vectors and matrices indicated in (17).

Next, we further consider the case that a large-scale MG which is governed by $m$ MASs. The hierarchical/cluster consensus algorithm provides a control solution for large-scale MAS, as illustrated in [30]. This control algorithm is suitable for flexible and scalable control of multiple MASs considering both intra MAS and inter MAS interactions. Without loss of generality, we simplify the representation by considering the number of agents in each group/MAS is the same. The proposed method is still applicable to the heterogeneous condition. Regarding the control framework shown in Fig. 2, the following feedback control protocol is proposed:

$$
\begin{aligned}
& {\left[\begin{array}{c}
u_{1} \\
u_{2} \\
\vdots \\
u_{m}
\end{array}\right]=-\underbrace{\left[\begin{array}{cccc}
K \tilde{L} & 0 & \cdots & 0 \\
0 & K \tilde{L} & \cdots & 0 \\
\vdots & \vdots & \ddots & \vdots \\
0 & 0 & \cdots & K \tilde{L}
\end{array}\right]\left[\begin{array}{c}
x_{1} \\
x_{2} \\
\vdots \\
x_{m}
\end{array}\right]}_{\text {Intra }-M A S}-\underbrace{\left[\begin{array}{cccc}
\hat{l}_{11} \Delta & \hat{l}_{12} \Delta & \cdots & \hat{l}_{1 m} \Delta \\
\hat{l}_{21} \Delta & \hat{l}_{22} \Delta & \cdots & \hat{l}_{2 m} \Delta \\
\vdots & \vdots & \ddots & \vdots \\
\hat{l}_{m 1} \Delta & \hat{l}_{m 2} \Delta & \cdots & \hat{l}_{m m} \Delta
\end{array}\right]\left[\begin{array}{c}
x_{1} \\
x_{2} \\
\vdots \\
x_{m}
\end{array}\right]}_{\text {Inter-MASs }}} \\
& +\underbrace{\left[\begin{array}{cccc}
K \tilde{G} & 0 & \cdots & 0 \\
0 & K \tilde{G} & \cdots & 0 \\
\vdots & \vdots & \ddots & \vdots \\
0 & 0 & \cdots & K \tilde{G}
\end{array}\right]\left[\begin{array}{c}
x^{r e f}-x_{1} \\
x^{\text {ref }}-x_{2} \\
\vdots \\
x^{\text {ref }}-x_{m}
\end{array}\right]}_{\text {Pinning Nodes }}
\end{aligned}
$$

where the matrix $\Delta \subseteq \mathbb{R}^{3 n \times 3 n}$ defines which agents have data exchange among each group. For simplicity, this paper considers $\Delta=K \tilde{G}$. The Laplacian matrix $\hat{L}=\left[\hat{l}_{i j}\right] \subseteq \mathbb{R}^{m \times m}$ indicates the interactions among groups. In compact form, (19) can be represented as

$$
\begin{aligned}
& U=-\left(I_{m} \otimes K \tilde{L}+\hat{L} \otimes \Delta\right) X+\left(I_{m} \otimes K \tilde{G}\right)\left(X^{r e f}-X\right) \\
& =\left(I_{m} \otimes K\right)\left[-\left(I_{m} \otimes \tilde{L}+\hat{L} \otimes \tilde{G}\right) X+\left(I_{m} \otimes \tilde{G}\right)\left(X^{r e f}-X\right)\right]
\end{aligned}
$$

where $U=\left[u_{1}, \ldots u_{m}\right]^{T}, X=\left[x_{1}, \ldots x_{m}\right]^{T}, X^{r e f}=1_{n \times 1} \otimes x^{r e f}$.

\section{Stability and Communication Delays}

Based on the problem formulation in preliminaries, the system dynamics are simplified into three first-order systems, 
i.e. $\dot{\omega}_{i}=u_{i}^{\omega}, \dot{V}_{i}=u_{i}^{V}$ and $\dot{p}=u_{i}^{p}$. Therefore, the closed-loop system with the proposed control protocol (20) is derived as:

$$
\dot{X}=\left(I_{m} \otimes K\right)\left[-\left(I_{m} \otimes \tilde{L}+\hat{L} \otimes \tilde{G}\right) X+\left(I_{m} \otimes \tilde{G}\right)\left(X^{r e f}-X\right)\right]
$$

Denoting the state error as $\delta=X-X^{\text {ref }}$, the following error system can be deduced:

$$
\dot{\delta}=-\left(I_{m} \otimes K\right)\left(I_{m} \otimes \tilde{L}+\hat{L} \otimes \tilde{G}+I_{m} \otimes \tilde{G}\right) \delta=-\Phi \delta
$$

By introducing the Lyapunov function $V(\delta)=\frac{1}{2} \delta^{T} \delta$, it can obtain that

$$
\dot{V}=\delta^{T} \dot{\delta}=-\delta^{T} \Phi \delta
$$

Therefore, the system stability criterion $\dot{V} \leq 0$ can be guaranteed when $\Phi \geq 0$. By LaSalle invariance principle, the system trajectories converge to the invariant set $S=\left\{\delta \in \mathbb{R}^{N} \mid \dot{V}=0\right\}$ [31]. By choosing control gains $k_{i}^{\omega}>0$, $k_{i}^{V}>0$, and $k_{i}^{p}>0$, the matrix $\left(I_{m} \otimes K\right)$ can be ensured to be positive definite. Based on the graph topology, the definite of matrix $\left(I_{m} \otimes \tilde{L}+\hat{L} \otimes \tilde{G}+I_{m} \otimes \tilde{G}\right) \quad$ can be examed by its eigenvalues. When $\left(I_{m} \otimes \tilde{L}+\hat{L} \otimes \tilde{G}+I_{m} \otimes \tilde{G}\right) \geq 0 \quad$ and $\left(I_{m} \otimes K\right)>0$, it implies that $\Phi \geq 0$, and thereby satisfying the stability criterion $\dot{V} \leq 0$.

In practical applications, the stability of the distributed control system is also impacted by the communications delay among the agents. Assuming the system as a network of integrator agents with equal communication time-delay $\tau>0$, $\tau \in\left(0, \tau^{*}\right)$ in all links. Based on Theorem 10 in [32], the upper boundary of tolerable communication delay can be estimated as a function of the largest eigenvalue of the Laplacian matrix:

$$
\tau^{*}=\pi / 2 \lambda_{\max }(\Phi)
$$

It is noted that for a given communications topology and choice of selected control gains there exists a tolerable delay $\tau<\tau^{*}$ that ensures the stability of the system. Similarly, for an observed time-delay $\tau>0$ and a given communications topology, there exists a combination of control gains $k_{i}^{\omega}, k_{i}^{V}$ and $k_{i}^{p}$ that ensure the stability of the system.

Considering the above fact, the following guideline for system design is proposed as follows:

Step 1: For a selected communications (graph) topology, ensure the matrix $\left(I_{m} \otimes \tilde{L}+\hat{L} \otimes \tilde{G}+I_{m} \otimes \tilde{G}\right) \geq 0$, such that Eq. (23) is satisfied.

Step 2: Choose control gains $k_{i}^{\omega}>0, k_{i}^{V}>0$, and $k_{i}^{p}>0$, considering the trade-off between tolerable communications delay and convergence speed.

Step 3: If required, fine-tuning the control gains for desired dynamic performance by means of time-domain simulations.

Remark 2: The proposed scalable distributed control method offers the following unique features:

1.The proposed method supports group plug-and-play functions. A group of agents and DGs can be plugged in and out by changing the inter group/MAS communication connections.
2. Each group/MAS can have control over its own communication graph and the number of DGs, which offers the operation flexibility for the owner of each group.

3 . The convergence and operational behaviour of the system can be adjusted by the grid operators by means of manipulating the communication links between the MASs.

Therefore, the proposed method provides a scalable and flexible way to manage MGs (e.g. number of DGs and convergence speed) for both DG owners and grid operators.

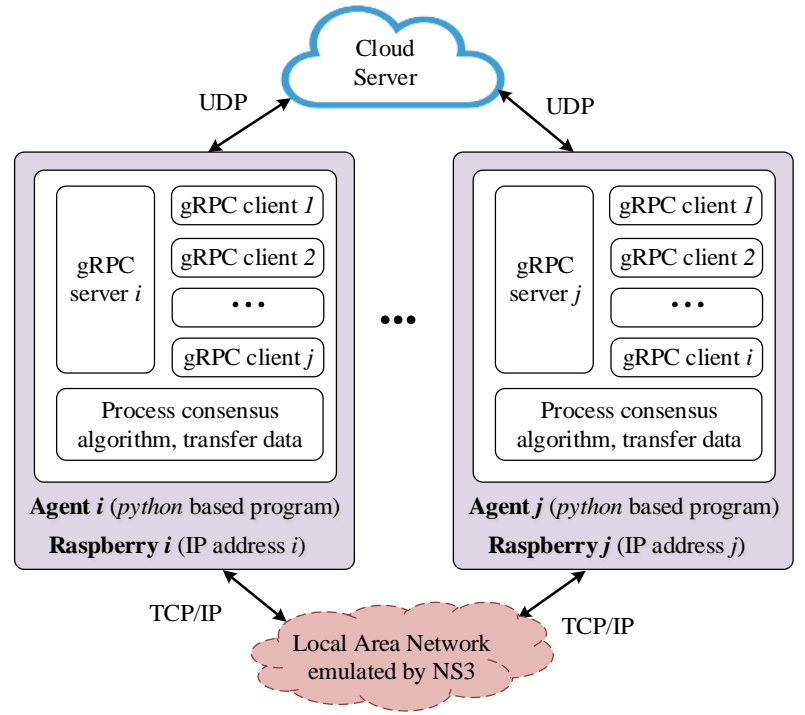

Fig. 3. The structure of each agent based on gRPC.

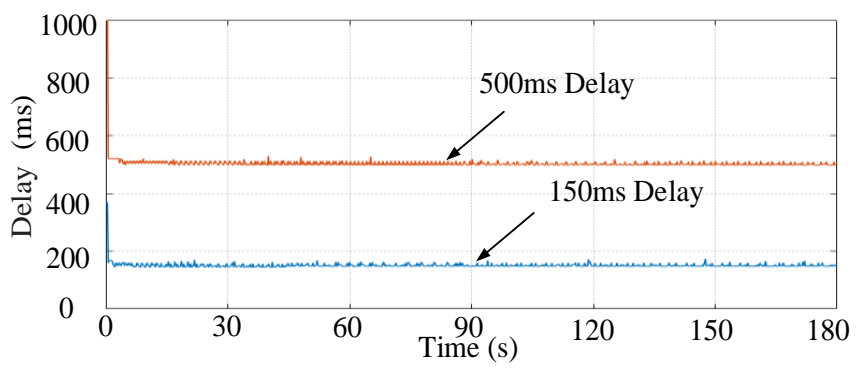

Fig. 4. Communication delay emulated by NS3 simulation tools.

\section{IMPLEMENTATION}

In this section, the experimental implementation of distributed secondary controllers on the MAS platform and its relationship with LAN and cloud server is introduced. Fig. 3 shows the structure of the MAS platform. The MAS is implemented in a cluster of RPis which is connected to the LAN through a network switch and cloud server through the Internet. The local communication is realized by TCP/IP protocol while the communication between MAS and cloud server is by user datagram protocol (UDP). An agent hosted in an RPi is a program written in pure python language. The communication among agents is in a client/server manner using gRPC and can be configured to any network topology. gRPC uses protocol buffers, which has a slightly simplified syntax for serializing structured data, for transferring messages. In gRPC based communication process, each agent is a server that waits for incoming messages and also can dispatch messages to corresponding method calls due to the fact that it is also a client 
of neighbor servers.

Each agent runs asynchronously in a RPi as an independent entity to manage a corresponding device in the physical layer. In every iteration, the consensus process in each agent implements the consensus control law in parallel and obtains data packets $\left\{\omega_{j}, V_{j}, m_{j}^{P} P_{j}\right\}$ from neighbors and $\left\{\omega_{i}, V_{i}, m_{i}^{P} P_{i}\right\}$ from the cloud server. The transferring data process in an agent is configured to ensure that only local information is exchanged with the cloud server and neighbourhood information is exchanged through the LAN. Then secondary control signals $\left\{\omega_{i}^{\text {nom }}, V_{i}^{\text {nom }}\right\}$ will be generated by each agent and uploaded to the cloud. The remote Opal-RT downloads the control signals, local controllers are designed to obtain data from corresponding agents and updates the electrical parameters periodically. Therefore the convergence of distributed implementation and its impact on the MG can be evaluated in a more realistic manner. Moreover, the disturbance of communication (i.e. latency, packet loss, cyber-attack, etc.) could be integrated directly to analyze the performance of the system. In this paper, the network delay is emulated by a network simulator tool 'NS3' [33], and the emulation results are shown in Fig. 4.

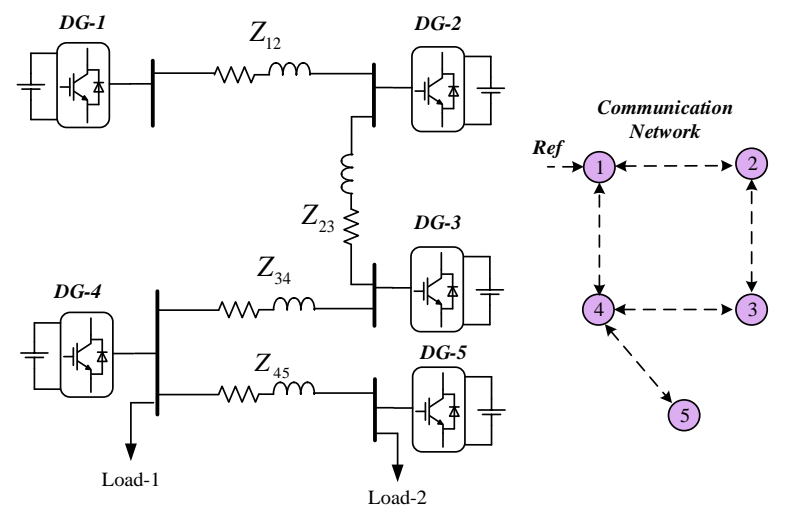

Fig. 5. Single line diagram of the 5-DG MG.

TABLE I

PARAMETERS OF THE MG TESTBED

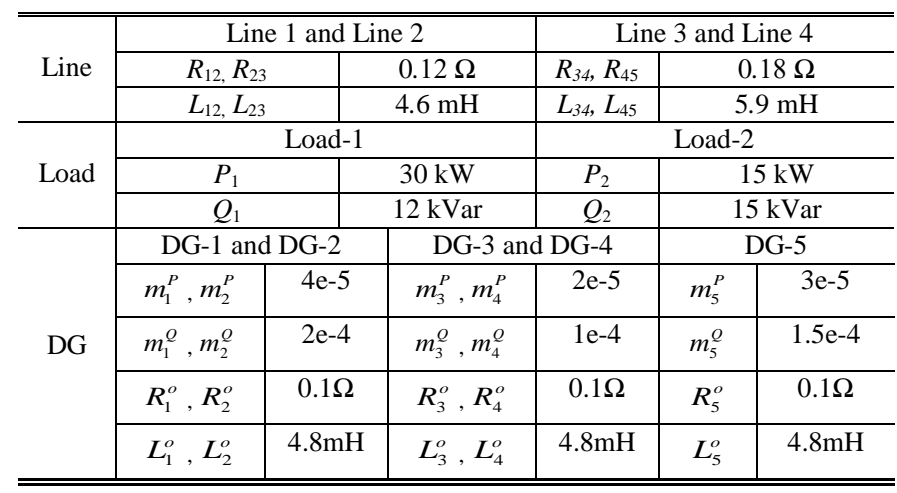

\section{HARDWARE-IN-THE-LOOP TEST RESULTS}

\section{A. Test Case Design}

The single line diagram of the MG and its associated communication topology is shown in Fig. 5, which is used in Case 1-3 and extended in Case 4-6. In the test system, inverterbased DGs are modeled using average models. The setup comprises three main parts: 1) the test MG in Opal-RT located at NTU, Singapore. 2) MASs on RPi cluster for distributed secondary control at University of Strathclyde, UK and UGA, France. 3) The cloud server on the Redis database as the interface between RPis and Opal-RT. The MG in Opal-RT is simulated at $50 \mu \mathrm{s}$ time-step. The data exchange rate among MG, cloud server and MASs is $10 \mathrm{~ms}$ via UDP and Internet. The inherent time-delay per trip is around $100 \mathrm{~ms}$. The communication rate of each MAS in LAN is also $10 \mathrm{~ms}$ by TCP/IP. The inherent time-delay in LAN is smaller than $10 \mathrm{~ms}$. The parameters of the MG testbed are listed in Table I. The parameters of secondary controllers are shown in Table II.

Seven test cases are considered and are organized as follows:

In Case 1-3, an islanded MG with 5 DGs has been set up to validate the proposed control framework. The control performance under normal condition, communication failures, and communication delays are demonstrated.

In Case 4-5, the MG is scaled up to 10 DGs and the MAS at UGA, France is also involved. The two groups of MASs interact with the proposed control via cloud service for remote control of MGs. A further test considering both real PV and load data is conducted in Case 5.

In Case 6, the group plug-and-play operation demonstrates the flexibility of the proposed method in a 15-DG MG, while the scalability for a large-scale MG system with 50 DGs is validated in Case 7.

TABLE II

PARAMETERS OF SECONDARY CONTROLLERS

\begin{tabular}{c|c}
\hline \hline $\begin{array}{c}\text { Frequency } \\
\text { Controller }\end{array}$ & $k_{1}^{\omega}=k_{2}^{\omega}=k_{3}^{\omega}=k_{4}^{\omega}=k_{5}^{\omega}=0.3$ \\
\hline $\begin{array}{c}\text { Voltage } \\
\text { Controller }\end{array}$ & $k_{1}^{V}=k_{2}^{V}=k_{3}^{V}=k_{4}^{V}=k_{5}^{V}=0.3$ \\
\hline $\begin{array}{c}\text { Power Sharing } \\
\text { Controller }\end{array}$ & $k_{1}^{P}=k_{2}^{P}=k_{3}^{P}=k_{4}^{P}=k_{5}^{P}=0.3$ \\
\hline Reference & $\omega^{\text {ref }}=50 \mathrm{~Hz}, V^{\text {ref }}=230 \sqrt{2} \mathrm{~V}$ \\
\hline
\end{tabular}

\section{B. Case 1: Validation of the Proposed Control Framework}

In Case 1, the effectiveness of the proposed distributed control for remote islanded MGs via cloud server is validated. Case 1 will serve as the base case for comparing the following cases with communication delays and failures. The simulation begins with Load-1 connecting to the MG at 0s. Load-2 is connected to the MG at 60 s, while Load-1 is disconnected from the $\mathrm{MG}$ at $120 \mathrm{~s}$. The $\mathrm{C}-\mathrm{HiL}$ experimental results of voltage, frequency, and the real power of each DG are shown in Figs. 6 (a)-(c). It can be observed in Figs. 6 (a) and (b) that the system frequency and voltage can be restored to the reference value effectively after the load changes. In Fig. 6(c), the real power is accurately shared among DG $1-5$ with the ratio of $3: 3: 6: 6: 4$, which is equal to the inverse ratio of droop coefficients. All the DGs in MG can autonomously change their power output to meet the load demand. The results of Case 1 prove that the distributed multi-agent control for remote MGs via cloud server is a valid method. 


\section{Case 2: Communication Failures}

In Case 2, the performance of the proposed control framework is validated under communication failures. The experimental events for this case are shown in Fig. 7. The distributed secondary controllers start to work at 10s. It is assumed that there is a failure of communication link 2-3 at 30s, and link 3-4 at 90s. The communication link 2-3 is reestablished at 150s. It should be noted that DG-3 loses its cyber connection from 90 s to 150 s, during which it is only governed by local droop control. Other conditions and control parameters remain the same as in Case 1. The C-HiL results for Case 2 are shown in Figs. 8 (a)-(c), and compared to the reference Case 1 in the following discussion. As observed, the communication failures do not severely influence the frequency/voltage restoration. However, as is expected, the real power sharing of DG-3 is not accurate due to the complete loss of cyberconnection.
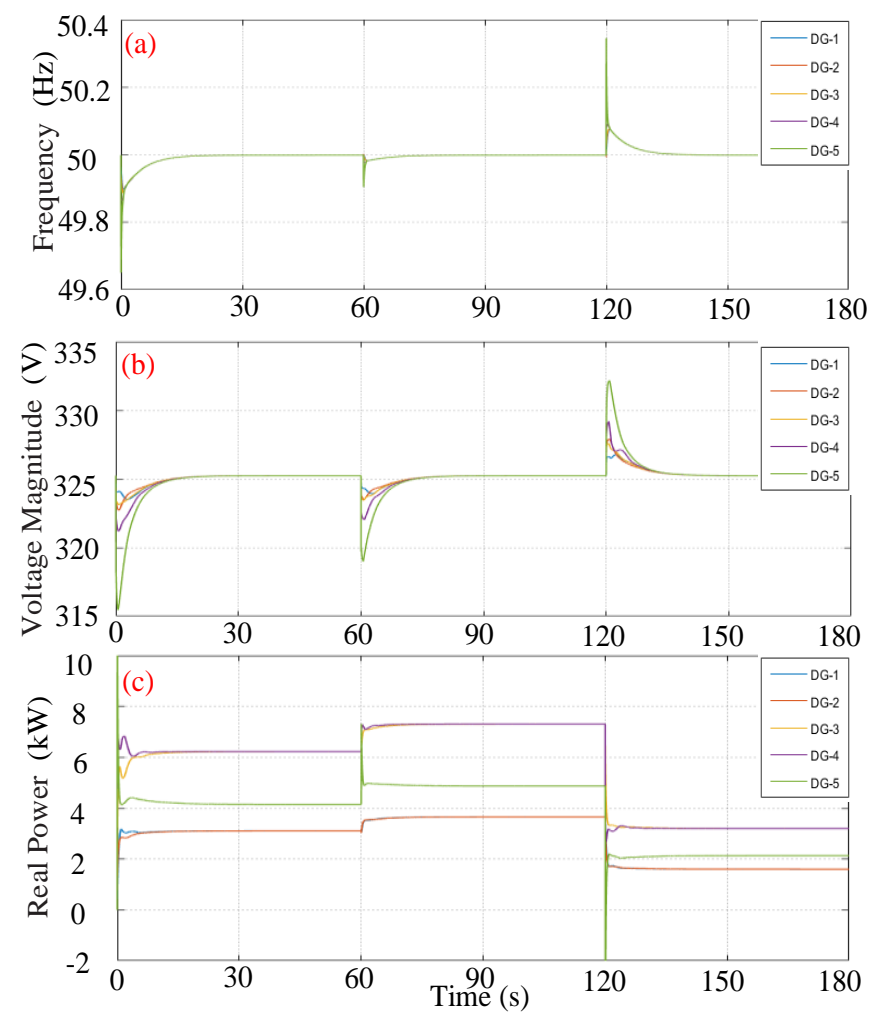

Fig. 6. The C-HiL results of frequency, voltage magnitude and the real power output of each DG in Case 1.

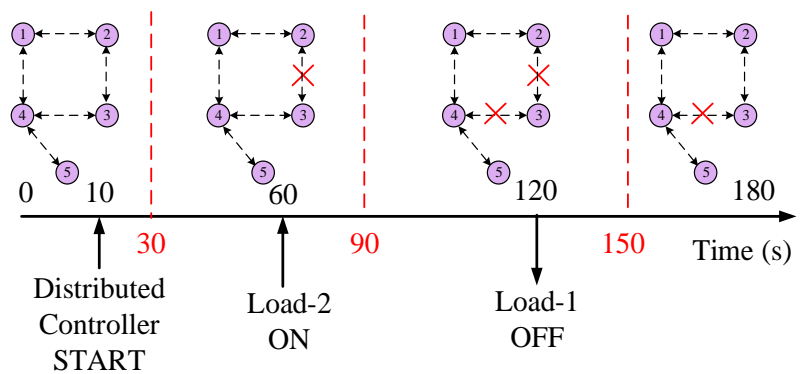

Fig. 7. Experimental events in Case 2.
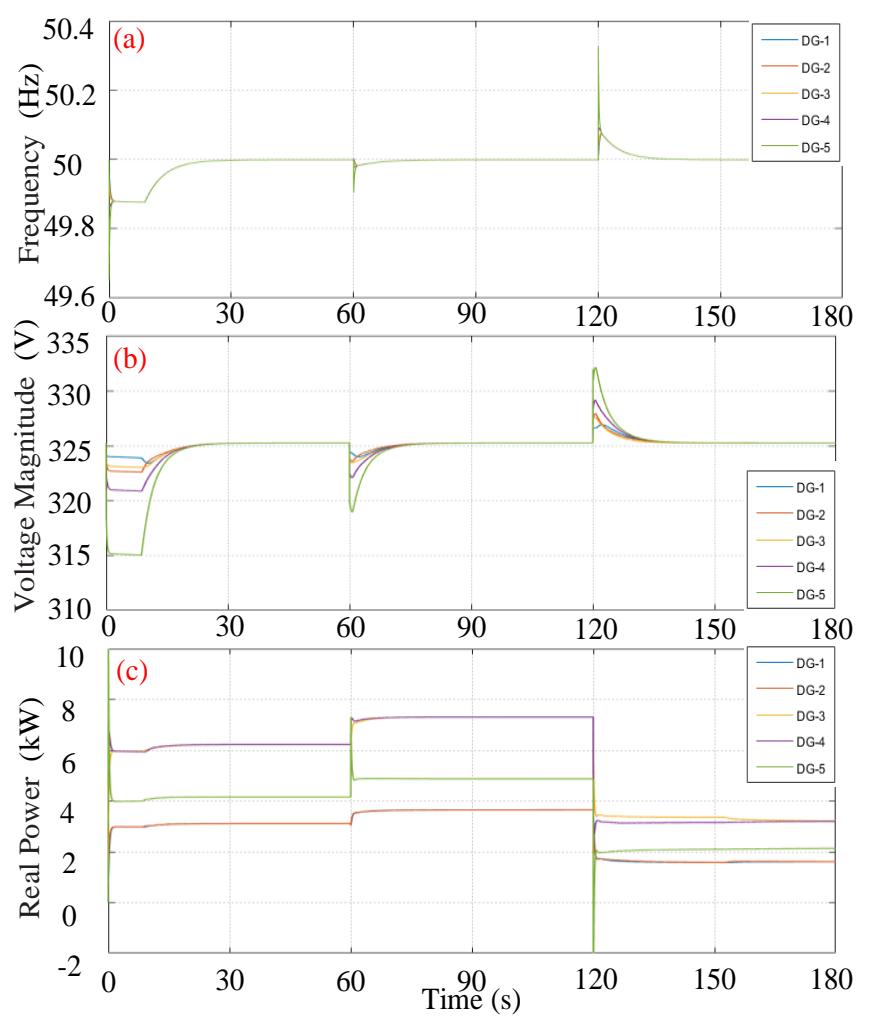

Fig. 8. The C-HiL results of frequency, voltage magnitude and the real power output of each DG in Case 1.

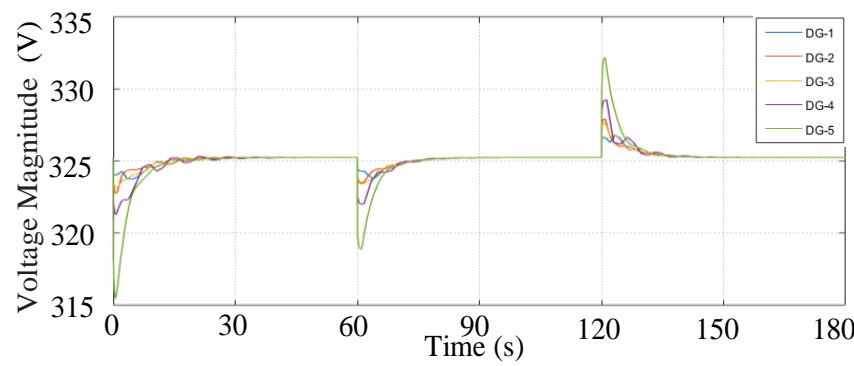

Fig. 9. The voltage of each DG with 500ms communication delay.

\section{Case 3: Communication Delays}

In Case 3, the performance of the proposed control framework with communication delays is evaluated. Here the communication delay refers to the delay between each of the DG agent. First, an experiment with 500ms delay is conducted, where the voltages of each of the DGs are shown in Fig. 9. As compared to the base case in Fig. 6(b), the voltage profiles exhibit small oscillations and slower speed of convergence. When the delay is increased to $1000 \mathrm{~ms}$, the MG frequency, voltage, and real power start to oscillate, as shown in Figs. 10 (a)-(c). The MG system is on the verge of instability. The largest eigenvalue of communication graph in Fig. 5 can be calculated as $\lambda_{\text {max }}=4.7226$ and the maximum tolerable delay as $\tau^{*}=\pi / 9.4452=0.3324 s$ from $(24)$ when all control gains are considered as 1. As $k_{i}^{V}=k_{i}^{\omega}=k_{i}^{P}=0.3$ in Cases 1-3, the convergence is guaranteed for all positive values of delay up to $\tau^{*}=1.108 \mathrm{~s}$. However, as is evident, the system performance is largely deteriorated when a delay of $1000 \mathrm{~ms}$ is considered. As shown in Fig. 11, after decreasing the control gain to 
$k_{i}^{V}=k_{i}^{\omega}=k_{i}^{P}=0.1$, the system becomes stable again. However, the convergence speed also decreases as per the trade-off relationship.
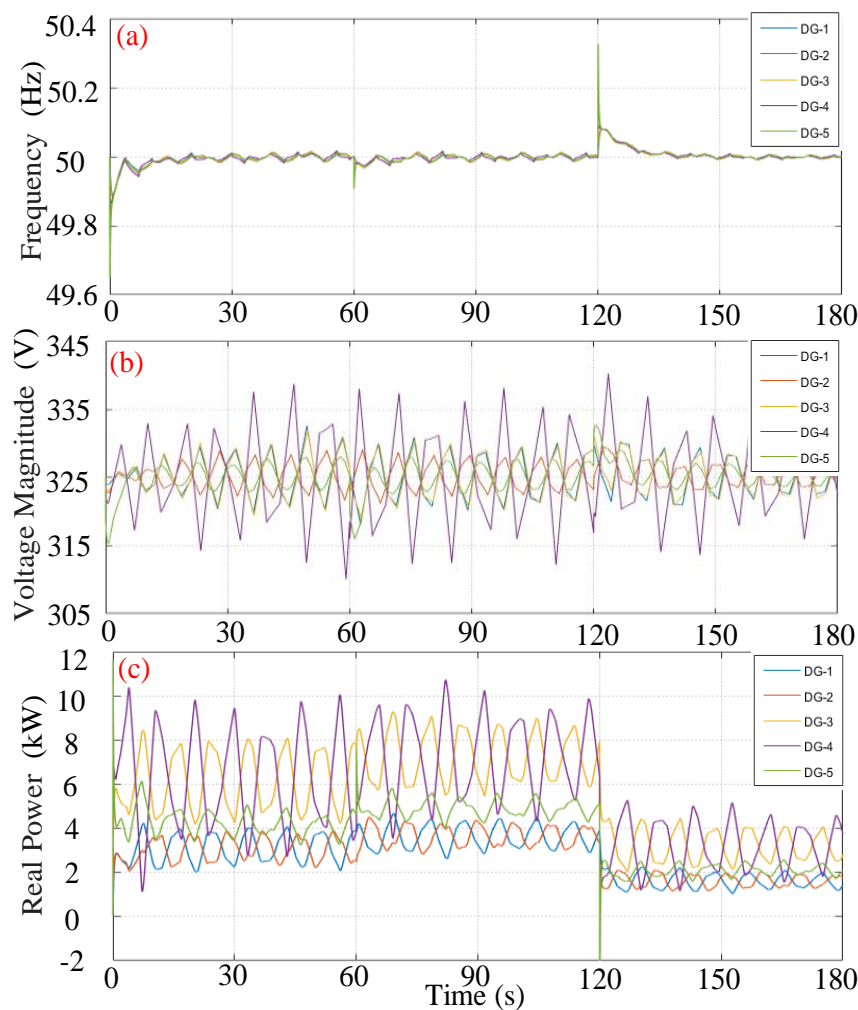

Fig. 10. The frequency, voltage and the real power of each DG with $1000 \mathrm{~ms}$ communication delay.

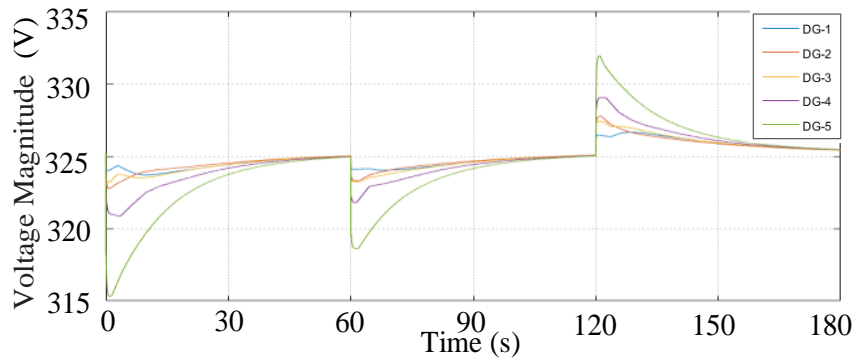

Fig. 11. The voltage of each DG with $1000 \mathrm{~ms}$ communication delay and small control gain.

\section{E. Case 4: Multi-Site Test}

In Case 4, the scalability of the proposed control framework is validated by the multi-site test. A cooperative C-HiL experiment interlinking three labs (NTU, Strathclyde, UGA) in different locations across the world has been conducted. The DG units in the MG are extended to 10 , and the load demand is increased to twice the initial loading in Table I. The communication graph of each MAS is the same as in Fig. 5, while the interaction among MAS is achieved by connecting agent 1 and 6 . The other parameters remain the same. The CHiL results for this case are shown in Figs. 12(a)-(c). In Figs. 12 (a) and (b), it can be found that the system frequency and voltage can be restored to the reference values effectively after the load changes. As can be observed from Fig. 12 (c), the real power is still accurately shared among DGs $1-10$ with the ratio of $3: 3: 6: 6: 4: 3: 3: 6: 6: 4$, according to their droop coefficients.
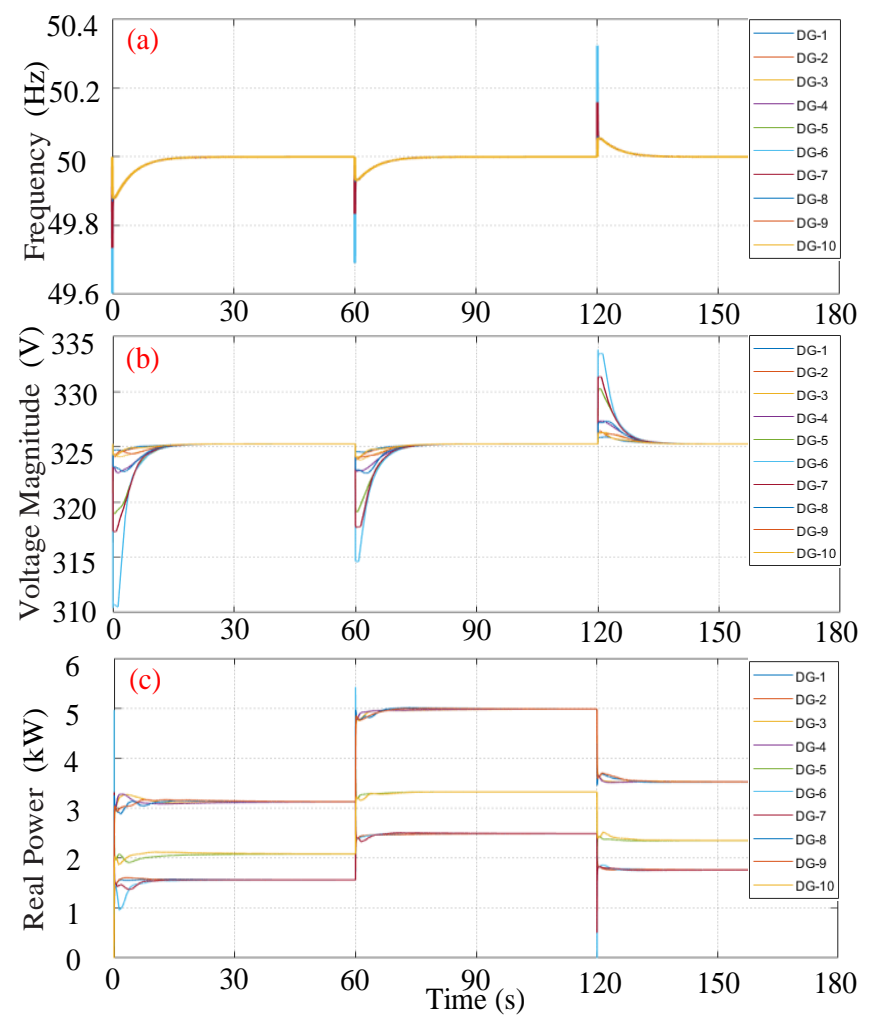

Fig. 12. The C-HiL results of frequency, voltage and the real power of each DG in Case 4.

\section{F. Case 5: Realistic PV and Load Data}

In Case 5, the proposed control framework is validated with real PV and load data, where the profiles are shown in Fig. 13. The other test conditions are the same as in Case 4. The PV data with one-second resolution measured by EPRI in June 2012 is used [34]. The C-HiL results for this case are shown in Figs. 14 (a)-(c). In Fig. 14 (a) and (b), it is found that even with PV and load fluctuations, the proposed control framework is able to regulate the frequency and voltage within a small variation range. The real power among DGs are shared according to their droop coefficients, i.e. 3:3:6:6:4:3:3:6:6:4 as shown in Fig. 14 (c). The results validate the effectiveness of the proposed method under practical conditions, i.e., with real PV and load profiles.

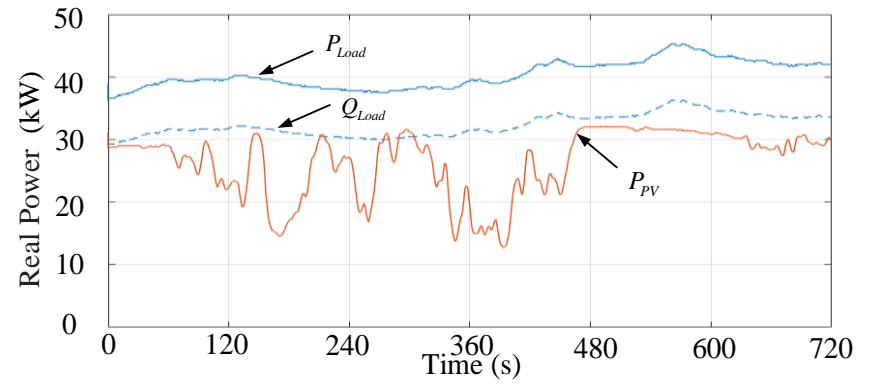

Fig. 13. The PV and load profiles used in Case 5. 

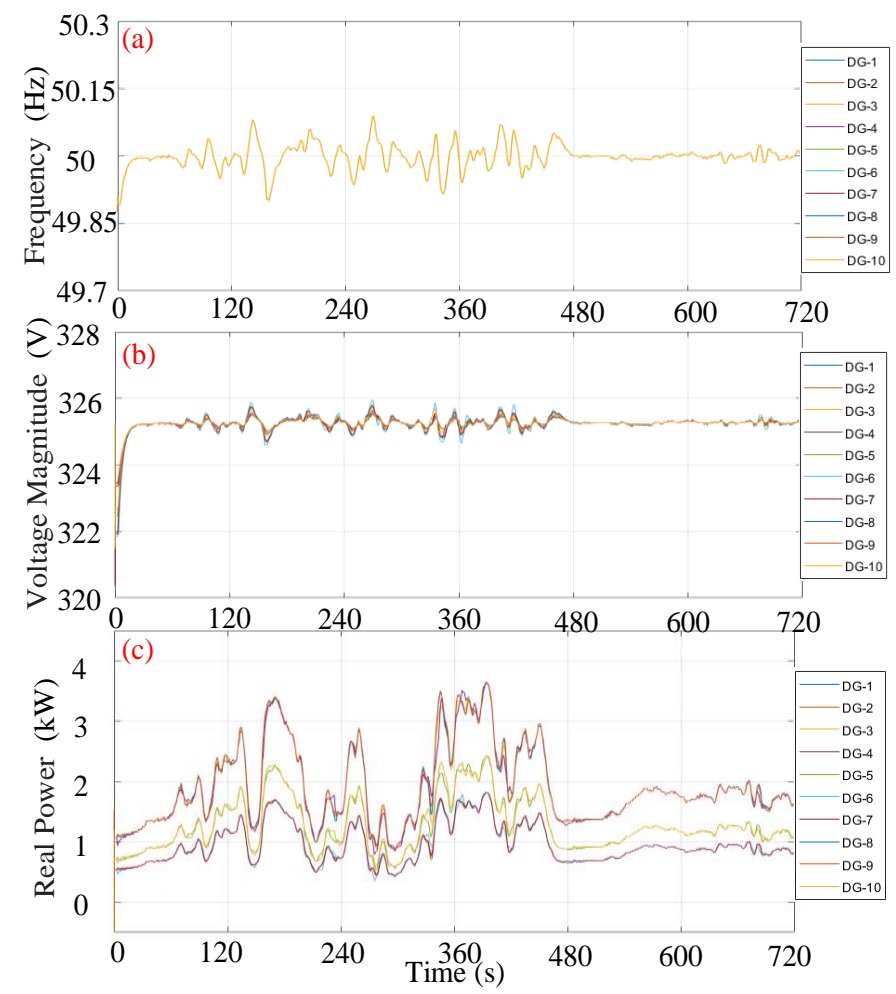

Fig. 14. The frequency of each DG in Case 5.

\section{G. Case 6: Group Plug-and-Play}

In Case 6, the proposed control framework is validated for group plug-and-play operation. The test case is to demonstrate the flexibility of the proposed controller for MGs governed by multiple MASs. In Case 6, it is considered that there are 15 DGs in the MG, which is controlled by 3 groups of MASs, as shown in Fig. 15. The interactions among groups can be managed by changing their communication links. The group plug-and-play events are shown on the top of Fig. 16, where both electrical and cyber systems are connected when there is a link between two groups. In Fig. 16, it can be observed that the power ratios (system states) will reach consensus with the proposed method when the groups are connected by links. The real power sharing among the DGs in this test case are shown in Fig. 17. In Fig. 18, the same electrical plug-and-play operations as shown in Fig. 16 are conducted, it can be observed that the group consensus cannot be reached without the considering group interactions.

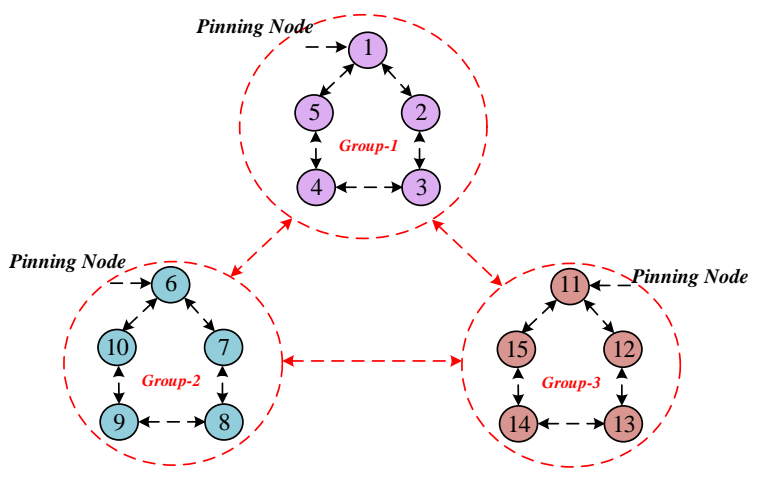

Fig. 15. A communication graph of 15 DGs managed by 3 groups of MASs.

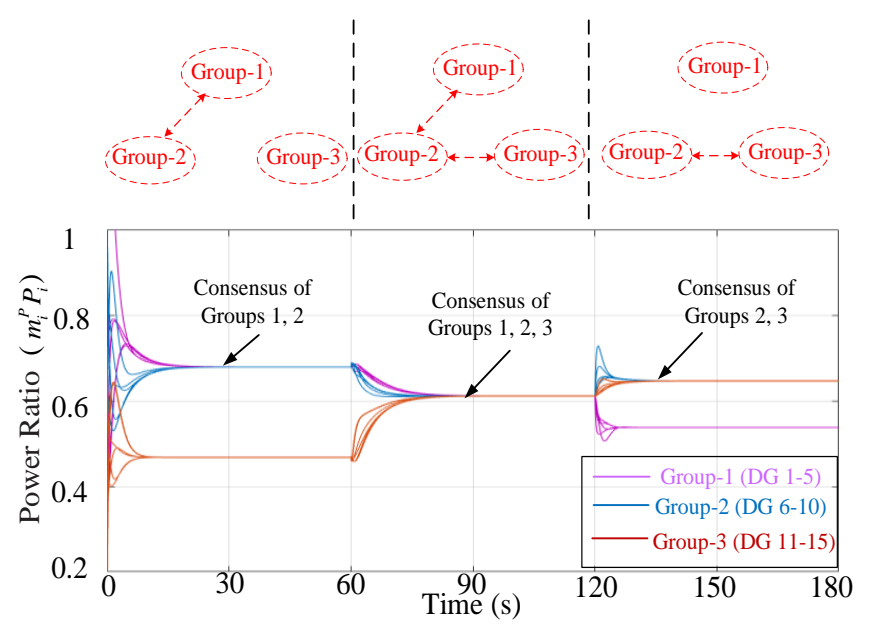

Fig. 16. The power sharing ratio $\left(m_{i}^{P} P_{i}\right)$ among each group (MAS) with the proposed method.

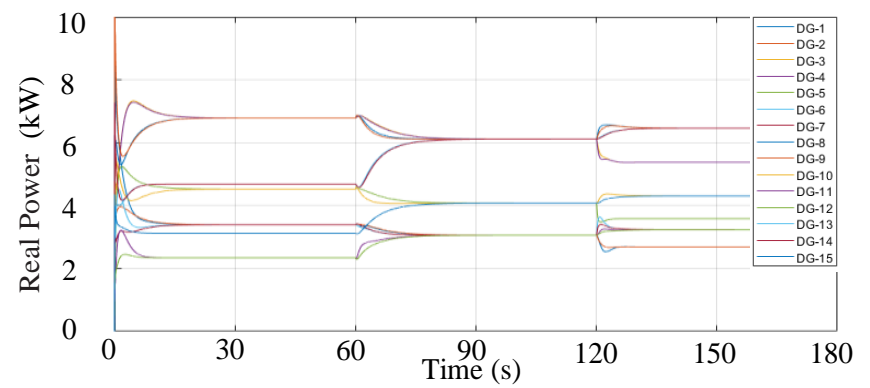

Fig. 17. The real power sharing of each DG in Case 6.

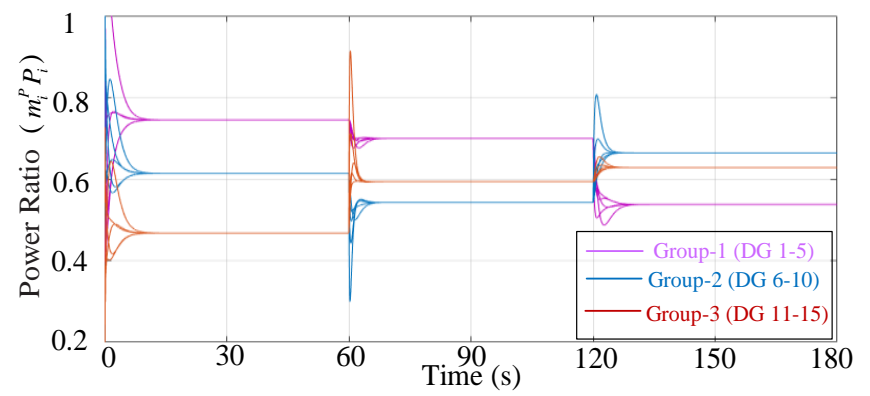

Fig. 18. The power sharing ratio $\left(m_{i}^{P} P_{i}\right)$ among each group (MAS) without group interactions.

Through the results in Case 6, it can be found that:

1. If the communication graph $\mathcal{G}$ is fully connected, then global consensus can be reached by all DGs. If the original communication graph $\mathcal{G}$ is partially connected, then the graph can be further divided into connected sub-graphs $\left\{\mathcal{G}_{1}, \mathcal{G}_{2}, \ldots\right\} \subseteq \mathcal{G}$. Local consensus will be reached within each sub-graphs.

2. The proposed method supports group plug-and-play functions by just managing the cyber connections among groups. The intra-group communication graphs are not influenced during the operation.

3. Compared to the proposed method, the methods without considering the group interactions (e.g [11]-[13], [26]) cannot address the scalable and flexible operation of MGs in Energy Internet effectively.

\section{H. Case 7: Application for Large-Scale Systems}

In Case 7, the proposed control framework is validated in a large-scale MG with 50 DGs. The test case is to demonstrate 
the scalability of the proposed control scheme. In Case 7, it is considered that the 50 DGs in the MG are governed by 10 groups of MASs. There is a Load-ON event at 60s and a LoadOFF event at 120s. The real power outputs of 50 DGs are shown in Fig. 19, where each DG shares the load demand according to its droop coefficient. The real power outputs of each sub-group are shown in Fig. 20, which indicates the power sharing among each group is the same. The system frequency and voltage magnitude can be still restored to their nominal values, as shown in Figs. 21 and 22. It validates the proposed method is capable for MG system with practically large number of DGs.

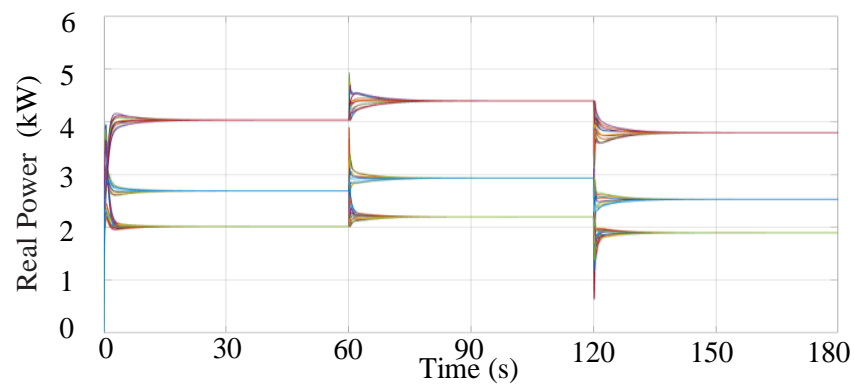

Fig. 19. The real power sharing of each DG in Case 7.

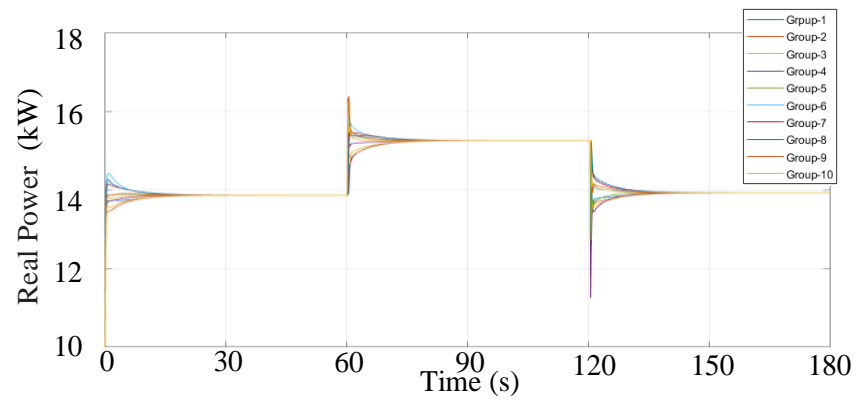

Fig. 20. The real power sharing among each sub-group in Case 7.

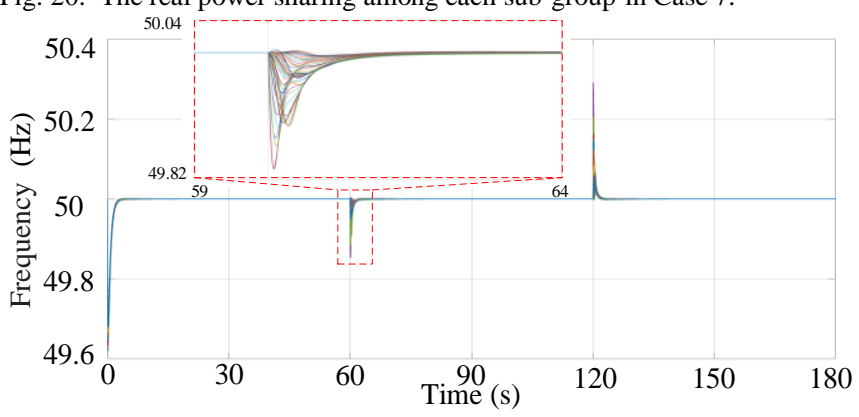

Fig. 21. The frequency of each DG in Case 7.

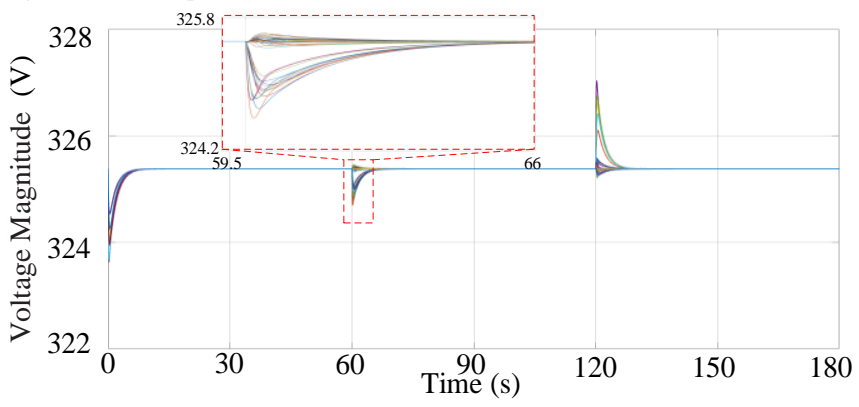

Fig. 22. The voltage magnitude of each DG in Case 7.

\section{CONCLUSIONS}

In this paper, a distributed control framework is proposed for the MG governed by different MAS groups. The proposed control law defines the data exchange within and among MASs to enable the flexible control of MG in Energy Internet. The distributed secondary control objectives are achieved with the evaluation of the stability considering network latency. The framework for multi-site implementation has been introduced and utilized collaboratively by NTU, Strathclyde, UGA to validate the proposed control framework. First, the experimental results show the control effectiveness under step load changes and communication failures. It has been shown that even with loss of 2 communication links, effectively isolating an entire DG, the proposed framework ensures stable operation of the MG. The capability of the control framework to tolerate delays of up to $1000 \mathrm{~ms}$ by tuning of the control gain in accordance with the proposed guidance is demonstrated. To establish its real-world applicability, the proposed method is evaluated in a 10-DG MG considering step load changes and real PV/load variations. The propsed method is able to regulate the voltage and frequency within $0.4 \%$, well within the operational requirements. Furthermore, the flexibility and scalability of the approach are demonstrated in MG with 15 DGs (3 MAS groups) and 50 DGs (10 MAS groups). Future work will focus on two important aspects: (i) the coordination of distributed secondary and tertiary control and (ii) communication graph management and optimization for robust and resilient control. (iii) control performance enhancement under imperfect communication such as packet loss.

\section{REFERENCES}

[1] K. K. Patel, and S. M. Patel, "Internet of things-IoT: definition, characteristics, architecture, enabling technologies, application \& future challenges," International Journal of Engineering Science and Computing, vol. 6, no .5, May, 2016.

[2] J. Cao, and M. Yang, "Energy internet--towards smart grid 2.0," 2013 Fourth International Conference on Networking and Distributed Computing. IEEE, 2013.

[3] K. Wang, et al. "A survey on energy internet: Architecture, approach, and emerging technologies," IEEE Systems Journal, vol. 12, no .3, pp. 24032416, Jan. 2017.

[4] N. Hatziargyriou, et al. "Microgrids," IEEE Power and Energy Magazine, vol. 5, no .4, pp. 78-94, Jul. 2007.

[5] J. M. Guerrero, et al. "Hierarchical control of droop-controlled AC and DC microgrids-A general approach toward standardization," IEEE Transactions on Industrial Electronics, vol. 58, no .1, pp. 158-172, Aug. 2010.

[6] Q. Shafiee, J. M. Guerrero, and J.C. Vasquez. "Distributed secondary control for islanded Microgrids-A novel approach," IEEE Transactions on Power Electronics, vol. 29, no .2, pp. 1018-1031, Feb. 2014.

[7] A. Bidram, F. L. Lewis, and A. Davoudi. "Distributed control systems for small-scale power networks: Using multiagent cooperative control theory," IEEE Control Systems, vol. 34, no .6, pp. 56-77, Dec. 2014.

[8] H. Qiu, B. Zhao, W. Gu and R. Bo, "Bi-Level Two-Stage Robust Optimal Scheduling for AC/DC Hybrid Multi-Microgrids," IEEE Transactions on Smart Grid, vol. 9, no. 5, pp. 5455-5466, Sept. 2018.

[9] C. Zhang, Y. Xu, Z. Y. Dong and L. F. Yang, "Multitimescale Coordinated Adaptive Robust Operation for Industrial Multienergy Microgrids With Load Allocation," IEEE Transactions on Industrial Informatics, vol. 16, no. 5, pp. 3051-3063, May 2020.

[10] D. K. Molzahn et al., "A Survey of Distributed Optimization and Control Algorithms for Electric Power Systems," IEEE Transactions on Smart Grid, vol. 8, no. 6, pp. 2941-2962, Nov. 2017.

[11] Y. Wang, et al. "Decentralised-distributed hybrid voltage regulation of power distribution networks based on power inverters," IET Generation, Transmission \& Distribution, vol. 13, no .3, pp. 444-451, Feb. 2019.

[12] X. Ge, F. Yang, and Q. Han, "Distributed networked control systems: A brief overview," Information Sciences, vol. 380, pp. 117-131, Feb. 2017.

[13] Y. Xu, et al. "Optimal distributed control for secondary frequency and voltage regulation in an islanded microgrid," IEEE Transactions on Industrial Informatics, vol. 15, no .1, pp. 225-235, Jan. 2019. 
[14] F. Guo, et al. "Distributed secondary voltage and frequency restoration control of droop-controlled inverter-based microgrids," IEEE Transactions on Industrial Electronics, vol. 62, no .7, pp. 4355-4364, 2015.

[15] M. Chen, X. Xiao, and J. M. Guerrero. "Secondary Restoration Control of Islanded Microgrids With a Decentralized Event-Triggered Strategy," IEEE Trans. Industrial Informatics, vol. 14, no .9, pp. 38703880, Sep. 2018.

[16] X. Liu, et al. "A Distributed Iterative Learning Framework for DC Microgrids: Current Sharing and Voltage Regulation," IEEE Transactions on Emerging Topics in Computational Intelligence, in press, 2018.

[17] E. A. A. Coelho, et al. "Small-signal analysis of the microgrid secondary control considering a communication time delay," IEEE Transactions on Industrial Electronics, vol. 63, no .10, pp. 6257-6269, Oct. 2016.

[18] R. Zhang and B. Hredzak, "Distributed Finite-Time Multiagent Control for DC Microgrids With Time Delays," IEEE Transactions on Smart Grid, vol. 10, no. 3, pp. 2692-2701, May 2019.

[19] Q. Sun, et al. "A multiagent-based consensus algorithm for distributed coordinated control of distributed generators in the energy internet," IEEE Transactions on Smart Grid, vol. 6, no .6, pp. 3006-3019, Nov. 2015.

[20] C. Dou, et al. "Multi-agent system-based event-triggered hybrid control scheme for energy Internet," IEEE Access, vol. 5, pp. 3263-3272, 2017.

[21] Y. Wang, M. H. Syed, E. Guillo-Sansano, Y. Xu and G. M. Burt, "Inverter-Based Voltage Control of Distribution Networks: A ThreeLevel Coordinated Method and Power Hardware-in-the-Loop Validation," IEEE Transactions on Sustainable Energy, in press, 2019.

[22] M. Stevic, et al. "Multi-site European framework for real-time cosimulation of power systems," IET Generation, Transmission \& Distribution, vol. 11, no. 17, pp. 4126-4135, 2017.

[23] B. Lundstrom, et al. "Trans-oceanic remote power hardware-in-the-loop: multi-site hardware, integrated controller, and electric network cosimulation," IET Generation, Transmission \& Distribution, vol. 11, no .18, pp. 4688-4701, 2017.

[24] P. Lin, et al. "A Semi-Consensus Strategy Toward Multi-Functional Hybrid Energy Storage System in DC Microgrids," IEEE Transactions on Energy Conversion, vol. 35, no. 1, pp. 336-346, March 2020.

[25] N. Pogaku, M. Prodanovic, and T. C. Green. "Modeling, analysis and testing of autonomous operation of an inverter-based Microgrid," IEEE Transactions on Power Electronics, vol. 22, no .2, pp. 613-625, Mar. 2007.

[26] A. Bidram, et al. "Secondary control of Microgrids based on distributed cooperative control of multi-agent systems," IET Generation, Transmission \& Distribution, vol. 7, no .8, pp. 822-831, 2013.

[27] C. W. Zhao, J. Jegatheesan, and S. C. Loon, "Exploring IoT application using raspberry pi," International Journal of Computer Networks and Applications, vol. 2, no .1, pp. 27-34, Jan. 2015.

[28] Google remote procedure call. [Online]. Available: https://grpc.io/.

[29] Redis website. [Online]. Available: https://redis.io/.

[30] Z. Duan, G. Zhai, and Z. Xiang, "State consensus for hierarchical multiagent dynamical systems with inter-layer communication time delay," Journal of the Franklin Institute, vol. 352, no .3, pp. 1235-1249, Mar. 2015.

[31] H. Zhang, F. L. Lewis, and Z. Qu. "Lyapunov, adaptive, and optimal design techniques for cooperative systems on directed communication graphs," IEEE Transactions on Industrial Electronics, vol 59, no .7, pp. 3026-3041, Jun. 2011.

[32] R. Olfati-Saber, and R. M. Murray, "Consensus problems in networks of agents with switching topology and time-delays," IEEE Transaction on Automatic Control, no. 49, vol .9, pp. 1520-1533, 2004.

[33] Network Simulator Tools NS3. [Online]. Available: https://www.nsnam.org/.

[34] 1-second resolution data, EPRI. [Online]. Available: http://dpv.epri.com/measurement_data.html.

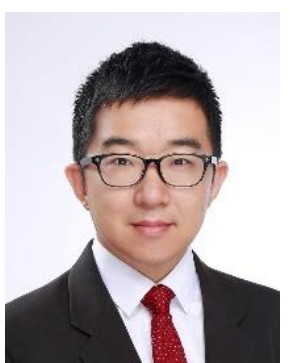

Yu Wang (S'12-M'17) received the B.Eng. degree in School of Electrical Engineering and Automation from Wuhan University, China in 2011, and the M.Sc. and Ph.D. degree from Nanyang Technological University, Singapore in 2012 and 2016, respectively. He is currently a research fellow in Nanyang Technological University, Singapore. $\mathrm{He}$ is leading and investigating industry projects in hybrid microgrid systems, energy storage systems, and cybersecurity of power systems. His research interests include distributed control and optimization in electrical power systems, microgrids and cyber-physical systems.

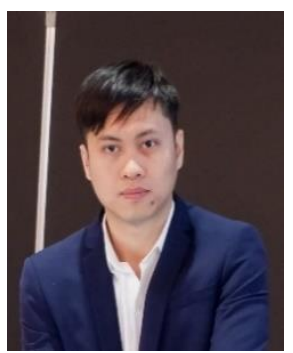

Tung-Lam Nguyen received the Master's degree in electrical engineering from National Taiwan University of Science and Technology, Taiwan, in 2014, and the Ph.D. degree from the University of Grenoble Alpes, Grenoble, France, in 2019. He is currently a Lecturer with the Faculty of Electrical Engineering, The University of Danang, University of Science and Technology, Danang, Vietnam, and a Post-Doctoral Researcher with the Grenoble Institute of Technology, Grenoble, France, as part of the G2ELab. His research interests include distributed control and optimization in microgrids and distribution networks, and co-simulation in smart grid.

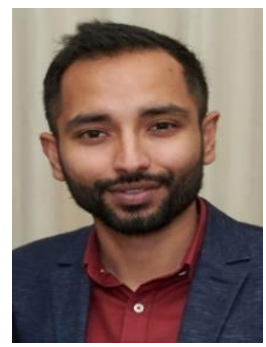

Mazheruddin H. Syed (S'11) received his BE degree in electrical and electronics engineering from Osmania University, India, his MSc degree in electrical power engineering from Masdar Institute of Science and Technology, UAE, and his PhD degree in electrical power systems from the University of Strathclyde, Glasgow, Scotland. Currently, he is a Research Fellow at the Institute for Energy and Environment, University of Strathclyde, Glasgow, Scotland and serves as the manager of the Dynamic Power Systems Laboratory microgrid. He is active in a number of national and international committees, has contributed to innovative power system research projects with a strong publication record. His research interests include demand side management, decentralized and distributed control, real-time controller and power hardware in the loop simulations, and systems level validations.

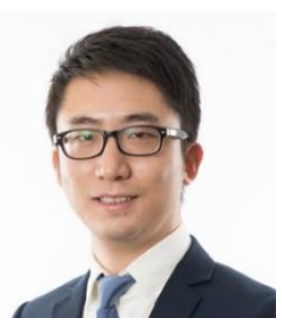

Yan Xu (S'10-M'13) received the B.E. and M.E degrees from South China University of Technology, Guangzhou, China in 2008 and 2011, respectively, and the Ph.D. degree from The University of Newcastle, Australia, in 2013. He is now the Nanyang Assistant Professor at School of Electrical and Electronic Engineering, Nanyang Technological University (NTU), and a Cluster Director at Energy Research Institute@ NTU (ERI@N), Singapore. Previously, he held The University of Sydney Postdoctoral Fellowship in Australia. His research interests include power system stability and control, microgrid, and dataanalytics for smart grid applications. $\mathrm{Dr} \mathrm{Xu}$ is an Editor for IEEE TRANSACTIONS ON SMART GRID, IEEE TRANSACTIONS ON POWER SySTEMS, IEEE POWER ENGINEERING LETTERS, CSEE Journal of Power and Energy Systems, and an Associate Editor for IET Generation, Transmission \& Distribution. 


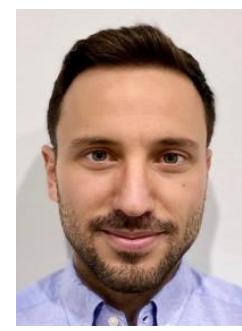

Efren Guillo-Sansano (S'13) received his M.Sc. and $\mathrm{PhD}$ degrees in Electrical Power Engineering from the University of Strathclyde, Glasgow, UK, in 2013 and 2018. He is currently a Research Associate with the Institute for Energy and Environment at the University of Strathclyde, Glasgow, UK, where he is a technical expert for the dynamic power system laboratory. His current research interests include the testing and validation of distributed energy resources and power electronics, power hardware in the loop, distributed control, and ancillary services.

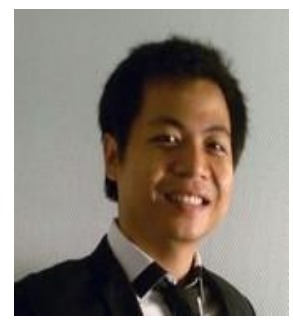

Van Hoa Nguyen received the M.S. degree and Engineering diploma in Mechatronics from Grenoble INP, France in 2010 and the Ph.D. degree from INSA Lyon, France in 2014. He is currently a researcher at National Institute of Solar Power (INES), French Alternative Energies and Atomic Energy Commission (CEA). His research interests include interoperability, holistic validation of cyber-physical energy system and hardware-in-theloop.

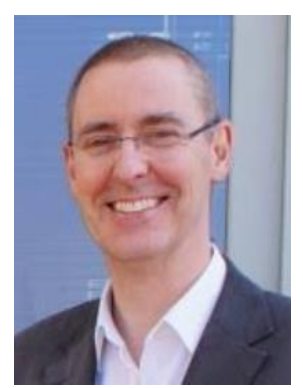

Graeme M. Burt (M'95) received the B.Eng. degree in electrical and electronic engineering, and the Ph.D. degree in fault diagnostics in power system networks from the University of Strathclyde, Glasgow, U.K., in 1988 and 1992, respectively. He is currently a Professor of electrical power systems at the University of Strathclyde where he co-directs the Institute for Energy and Environment, directs the Rolls-Royce University Technology Centre in Electrical Power Systems, and is lead academic for the Power Networks Demonstration Centre (PNDC). In addition, he serves as spokesperson for the board of DERlab e.V., the association of distributed energy laboratories. His research interests include the areas of power system protection and control, distributed energy, and experimental validation.

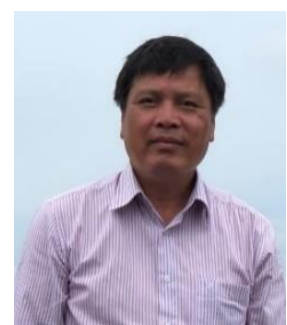

Quoc-Tuan Tran received his Ph.D. degree in Electrical Engineering and Habilitation à Diriger des Recherches degree from the Grenoble Institute of Technology in 1993 and 2000, respectively. He is an IEEE senior member. He is currently a Professor with INSTN - Paris Saclay University, and a Scientific Manager with Alternative Energies and Atomic Energy Commission (CEA) - National Institute for Solar Energy (INES). His research interests are in the fields of power system analysis, operations, electromagnetic transients, distributed generation, smart-grid and renewable energy.

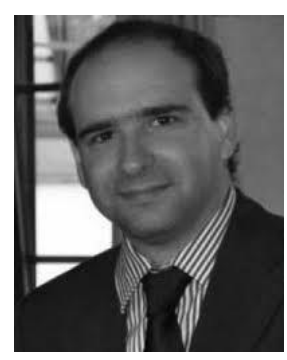

Raphael Caire received the Diplôme d'Etudes Approfondies and Doctorat de l'INPG degrees from the Institut National Polytechnique de Grenoble in 2000 and 2004, respectively. He has been with the Power Electronics field at the Center of Power Electronic System (CPES), USA, since 2000 and within several EDF research centers in Germany and France from 2004 to 2006 . He is currently an Associate Professor with the Grenoble Institute of Technology, Ecole d'ingénieurs en énergie eau et environnement, Grenoble Electrical Engineering Laboratory. His research is centered on the impacts, production control of dispersed generation on distribution system and critical infrastructures. 